\title{
Research of the Measurement on Palmitic Acid in Edible Oils by Near-Infrared Spectroscopy
}

\author{
Hui Li, Jingzhu $\mathrm{Wu}^{*}$, and Cuiling Liu \\ College of Computer \& Information Engineering, Beijing Technology and Business University, \\ Fucheng Road 33, 100048 Beijing, P. R. China \\ pubwua163. com
}

\begin{abstract}
A method for determination of palmitic acid in edible oils by the near-infrared spectroscopy was addressed in this paper. 56 samples were collected in the experiment. In terms of concentration content gradient method, 44 samples were selected for modeling set and 12 for testing set. This paper described the utilization of PLS for establishing a quantitative analysis model for predicting the content of palmitic acid in edible oils by near-infrared spectroscopy. The result shows that the model has a high accuracy for predicting the palmitic acid content with vector normalization and first-derivative preprocessing spectra with its best main factorial number of 8.The determination coefficients (R2), root mean square error of cross validation (RMSECV), root mean square error of prediction (RMSEP) and average bias are 99.59, 0.162, 0.306 and 0.148 , respectively. The model used in the paper can be adopted for the measurement of palmitic acid in edible oils accurately.
\end{abstract}

Keywords: Near-infrared Spectroscopy, Palmitic Acid, Edible Oils, quantitative analysis.

\section{Introduction}

The near-infrared reflectance is a kind of electromagnetic wave whose wavelength range is between $780 \mathrm{~nm}$ and $2526 \mathrm{~nm}$. It is the earliest non visible light founded by people. The spectrum is produced by frequency doubling of vibration at fundamental and frequency composition absorbing in middle infrared. The compounds contain X$\mathrm{H}$ groups which make the analysis possible. Near Infrared spectroscopy is one of the most rapidly developing detection technology. It has the characteristics of fast, accurate, non-polluting and non-destructive. With the improving of hardware and software of near-infrared (NIR), the applying area of NIR must be enlarged rapidly. It is widely founded that the edible oils are often adulterated with some other inferior quality materials. A fast and accurate detection method to determine the content of the major constituent of edible oils is extremely urgent.

Palmitic acid is an important index of the quality of edible oil. The content of it affects the quality of oils directly. For this reason, a method for the determination of palmitic acid in edible oils by the near infrared spectroscopy is addressed in this

\footnotetext{
* Corresponding author.
} 
paper. It also established a more accurate prediction model and thereby fulfills the fast detection of palmitic acid content.

\section{Apparatus and Materials}

\subsection{Apparatus and Spectrum Determination}

Use MPA in the agricultural institute to collect spectrum and fiber optics probe to scan the samples. Path length is $2 \mathrm{~mm}$. The resolution is $8 \mathrm{~cm}^{-1}$ and the number of scan times is 32 . The range of spectrum is between $12500 \mathrm{~cm}^{-1}$ and $4000 \mathrm{~cm}^{-1}$. Software OPUS 6.5 is adopted to establish the analytic model.

\subsection{Materials}

There are 56 edible oils which come from different brands and batches in the experiment including peanut oil, corn oil, palm oil, olive oil and soybean oil. In terms of concentration content gradient method, 44 samples are selected for modeling set and 12 for testing set in different palmitic concentration range. The actual palmitic content is measured by the national standard method. The relationship between the amount of modeling set samples and chemical value distribution range of palmitic is shown in Figure 1.

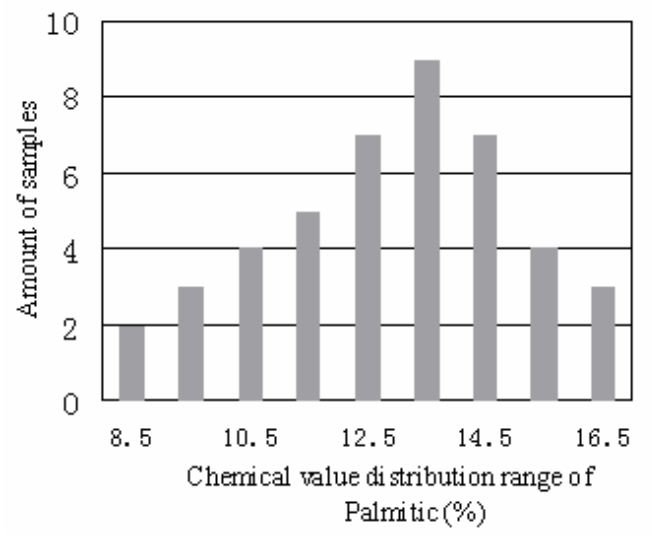

Fig. 1. Chemical value distribution range of palmitic

\subsection{NIR Scanning}

All the samples are without any chemical treatment before experiment. Put the fiber optics into the bottles which content the oils and scan them in sequence. In order to avoid cross contamination, petroleum ether is used to clean the fiber optics before every scanning. The NIR spectrograms of samples are shown in Figure 2. 


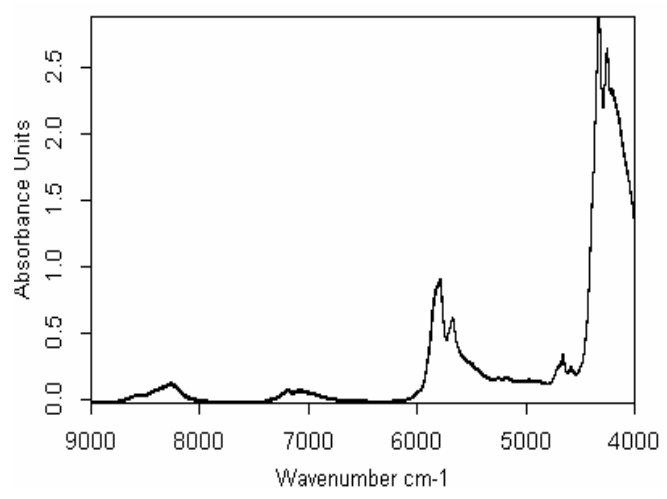

Fig. 2. Near infrared spectrogram of edible oil samples

\section{Results and Discussion}

\subsection{Choosing of the Best Main Factorial Number}

The main factorial number affects the predictive accuracy of the model. An excessive number will lead to over-fit phenomena. Conversely, a too small one will cause lack of fit. By implication, the model has a high accuracy forecast precision when the factorial number is 8 . The relationship between main factorial number and RMSECV is shown in Figure 3.

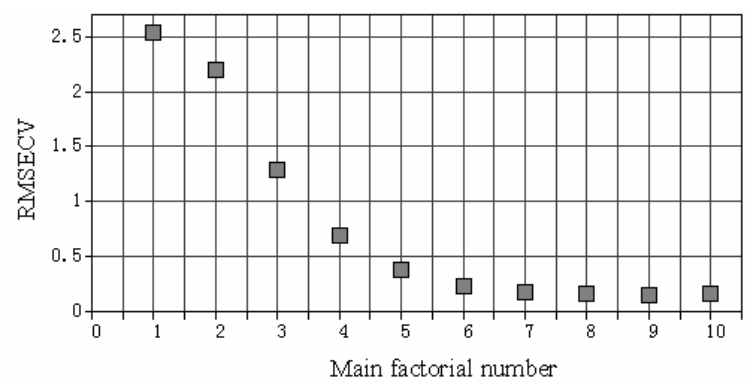

Fig. 3. Relationship between main factorial number and RMSECV

\subsection{Choosing of the Spectrum Preprocessing Method}

In the experiment, it gets the information of spectrum by scanning the full waveband NIR and describes the utilization of PLS for establishing a quantitative analysis model for predicting the content of palmitic acid in edible oils by using software of OPUS. After optimization analyzing, the determination coefficients $\left(\mathrm{R}^{2}\right)$ and root mean square error of cross validation (RMSECV) are both at low forecast precision by using first derivative, second derivative, multiplicative scattering correction and vector normalization. However, the preprocessing method of first derivative with vector 
normalization can get a high accuracy model. $\mathrm{R}^{2}$ and RMSECV are 99.59 and 0.162 , respectively. The result is shown in Figure 4.

\subsection{Analysis of Unknown Samples}

In the range of wavelength $12492.9-7498.1 \mathrm{~cm}^{-1}$ and $6101.8-4246 \mathrm{~cm}^{-1}$.The 12 samples are used to test the prediction model. $\mathrm{R}^{2}$ is 99.1 and RMSEP is 0.306 . The

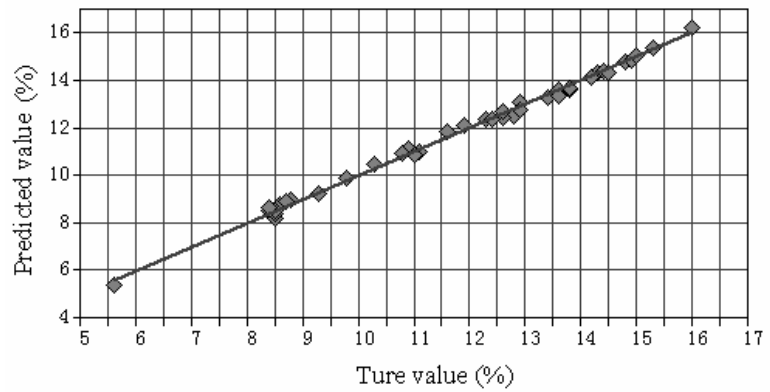

Fig. 4. Optimized model of calibration with partial least squares (PLS)

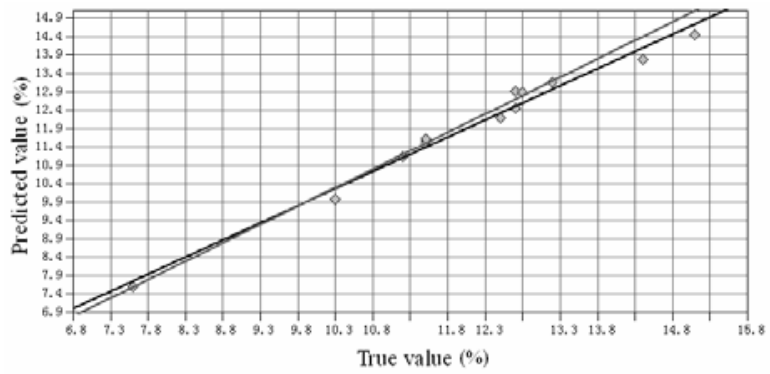

Fig. 5. Predicted and true value of validation with the model

Table 1. True value and predicted value of the 12 samples

\begin{tabular}{ccc}
\hline Sample number & True value $(\%)$ & Predicted value $(\%)$ \\
\hline 46 & 14.4 & 13.785 \\
47 & 11.2 & 11.126 \\
48 & 11.5 & 11.552 \\
50 & 11.5 & 11.621 \\
52 & 10.3 & 9.983 \\
53 & 7.6 & 7.601 \\
55 & 12.7 & 12.903 \\
56 & 13.2 & 13.161 \\
57 & 15.1 & 14.461 \\
58 & 12.8 & 12.898 \\
59 & 12.7 & 12.462 \\
60 & 12.5 & 12.174 \\
\hline
\end{tabular}


relationship between predicted value and true value is shown in Figure 5.The angle between prediction curve and standard curve is small. The predicted value converges towards the true value and the samples points distribution on both sides of the curve closely. There are no samples beyond the range of the model and so that it has a general applicability.

The predicted and true value of the 12 testing set samples are shown in Table 1.

\section{Conclusion}

(1). In the wavelength range of $12492.9-7498 \mathrm{~cm}^{-1}$ and $6101.8-4246 \mathrm{~cm}^{-1}$, the model has a high accuracy for predicting the palmitic acid content with vector normalization and first-derivative preprocessing spectra. 44 samples are selected for modeling set. The best main factorial number, $\mathrm{R}^{2}$ and RMSECV are 8, 99.59 and 0.162 , respectively. Use this model to test the 12 samples. $\mathrm{R}^{2}$, RMSEP and average bias are 99.1, 0.306 and 0.148 , respectively.

(2). It is plausible to use NIR spectroscopy to test the content of palmitic in edible oils. However, this experiment is still at the initial stage. Regarding to the other important components in edible oils, the model still needs further improvement so as to get more generalization and application.

Acknowledgments. This work is supported by superior and refined talents of Beijing funding project No. 20081D0500300130. The authors would like to thank Professor Xiaochao Zhang from Chinese Academy of Agricultural Mechanization Sciences to supply the NI Spectrograph to help us collect samples' spectrum.

\section{References}

1. Christie, O.H.J.: Data Laundering by Target Rotation in Chemistry-based Oil Exploration. J. Chemometr 10, 453-461 (1996)

2. Lu, W.Z., Yuan, H.F., Xu, G.T.: Modern Near Infrared Spectroscopy Analysis Technique, China. Petroleum Press, Beijing (2000)

3. Cui, X.J., Yuan, C.M., Xu, L.H.: Near-Infrared Spectroscopic Analysis of Peroxides in Peanut Oil. Chinese Journal of Applied Chemistry 25(3), 375-377 (2008)

4. Yu, Y.B., Zang, P.: The Rapid Analysis of Fatty Acids in Vegetable Oils by Near Infrared Spectrum. Spectroscopy and Spectral Analysis 28(7), 1554-1558 (2008)

5. Kvalheim, O.M.: Interpretation of Latent Variable Regression Models. Chemometrics and Intelligent Laboratory Systems 7, 39-51 (1989)

6. Zhang, D.Q., Chen, X.N., Sun, S.Q.: The Quantified Analysis of Fresh Mutton Tenderness Using PLS Methods and Fourier Transform Near Infrared Spectroscopy. Spectroscopy and Spectral Analysis 28(11), 2550-2553 (2008)

7. Mcshane, M.J., Cote, G.L., Spiegelman, C.H.: Assessment of Partial Least-Squares Calibration and Wavelength Selection for Complex Near-infrared Spectra. Applied Spectrpscopy 52(6), 878-884 (1985) 